\begin{tabular}{|c|l|}
\hline Title & $\begin{array}{l}\text { Nonequilibrium Quasiparticle Dynamics in Bi-Based Superconductors Measured by Modulation Photoexcitation } \\
\text { Spectroscopy }\end{array}$ \\
\hline Author(s) & Toda, Y.; Mochizuki, H.; T suchiya, S.; Kurosawa, T.; Oda, M.; Mertelj, T.; Mihail ovic, D. \\
\hline Citation & $\begin{array}{l}\text { Journal of superconductivity and novel magnetism, 31(3), 753-756 } \\
\text { https://doi.org/10.1007/310948-017-4325-9 }\end{array}$ \\
\hline Issue Date & 2018-03 \\
\hline Doc URL & http://hdl.handle.net/2115/72702 \\
\hline Rights & The final publication is available at link.springer.com \\
\hline Type & article (author version) \\
\hline File Information & SuperstripesY V 2.pdf \\
\hline
\end{tabular}

Instructions for use 


\title{
Nonequilibrium quasiparticle dynamics in Bi-based superconductors measured by modulation photoexcitation spectroscopy
}

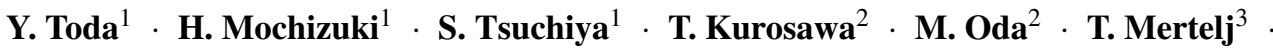 \\ D. Mihailovic ${ }^{3}$
}

Received: 30 August 2017 / Accepted: 5 September 2017

\begin{abstract}
We develop an orbital angular momentum (OAM) excitation pump-probe $(\mathrm{Pp})$ spectroscopy and perform experiments on $\mathrm{a}_{2} \mathrm{Sr}_{2} \mathrm{CaCu}_{2} \mathrm{O}_{8+\delta}(\mathrm{Bi} 2212)$ superconductor. The OAM modulation for the pump is realized by using a transverse mode conversion technique which allows to modulate the chirality of the optical OAM with a fixed polarization. The result reveals a difference of the OAM dependent transient reflectivity changes below $T_{\mathrm{c}}$, indicating that the pump OAM can break the chiral symmetry of the superconducting (SC) state. The OAM dependences of the transient signal imply that the quasi-particle $(\mathrm{QP})$ excitation with OAM enhances the SC with opposite chirality and/or suppress the SC with equal chirality. The temperature dependence of the OAM dynamics shows virtually no pairing fluctuation that usually appears in the QP dynamics observed by conventional Pp, suggesting that the nonequilibrium OAM dynamics is sensitive to the delocalized OAM associated with long range order.
\end{abstract}

Keywords high- $T_{\mathrm{c}}$ cuprate superconductor $\cdot$ time-resolved spectroscopy $\cdot$ orbital angular momentum $\cdot$ optical vortex

This work is supported by KAKENHI of JSPS (16H03876).

Y. Toda $\cdot$ H. Mochizuki $\cdot$ S. Tsuchiya

Department of Applied Physics, Hokkaido University, Kita-13, Nishi-

8, Kita-ku, Sapporo 060-8628, Japan

Tel.: +81-11-706-6627

Fax: +81-11-706-6627

E-mail: toda@eng.hokudai.ac.jp

T. Kurosawa $\cdot$ M. Oda

Department of Physics, Hokkaido University, Kita-10 Nishi-8 Kita-ku, Sapporo 060-0810, Japan

T. Mertelj · D. Mihailovic

Complex Matter Department, Jozef Stefan Institute, Ljubljana, SI1000, Slovenia

\section{Introduction}

The symmetry analysis in high- $T_{\mathrm{c}}$ superconductors is quite important for understanding the mechanism of the superconductivity, and has been performed using various techniques [1,2]. For example, scanning tunneling microscopy /spectroscopy studies have indicated the presence of charge-order associated with the pseudogap formation in $\mathrm{Bi}_{2} \mathrm{Sr}_{2} \mathrm{CaCu}_{2} \mathrm{O}_{8+\delta}$ (Bi2212) [3-7], which is quite different from the static stripe-order observed in $\mathrm{La}_{2-x} \mathrm{Ba}_{x} \mathrm{CuO}_{4}$ (LBCO) and $\mathrm{La}_{1.6-x} \mathrm{Nd}_{0.4} \mathrm{Sr}_{x} \mathrm{CuO}_{4}$ (LNSCO) by neutron scattering studies[8,9]. Moreover, gyrotropy appears at the same temperature as stripe order in La-family, suggesting the rotation of the stripe order with broken chiral symmetry $[10,11]$.

Polarized optical pump-probe (Pp) spectroscopy using near-infrared/visible femtosecond pulse laser is also a useful technique for the symmetry detection. In our previous study, the $\mathrm{B}_{1 \mathrm{~g}}$ and $\mathrm{B}_{2 \mathrm{~g}}$ symmetry breaking of the transient reflectivity changes $(\Delta R)$ have been observed below $T^{*}$ in Bi2212[12]. Here the high energy pump-excited carriers on a short time scale result in totally symmetric excitation of quasiparticles (QPs) that reveal the polarization anisotropy of $\Delta R$ responsible for the symmetry breaking. The symmetry analysis is thus realized by the polarization dependence of the time-delayed probe pulse.

Based on the polarization symmetry analysis above, in this work, we develop a novel orbital angular momentum (OAM) modulation Pp spectroscopy for the global symmetry analysis. Here we newly introduce the optical vortex pulses for the excitation. The optical vortex carries an OAM defined by $\ell \hbar$ per photon[13], where $\ell$ indicates the azimuthal phase index of the transverse mode of the beam and corresponds to the winding number of helical wavefront. The sign of $\ell$ specifies the chirality of the vortex. We perform the OAM-resolved Pp spectroscopy on an underdoped (UD) 
(a)

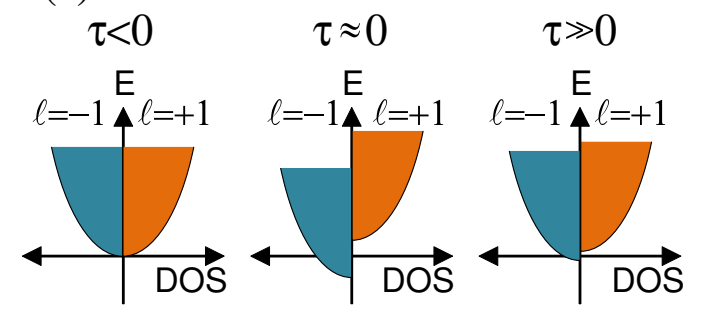

(b)

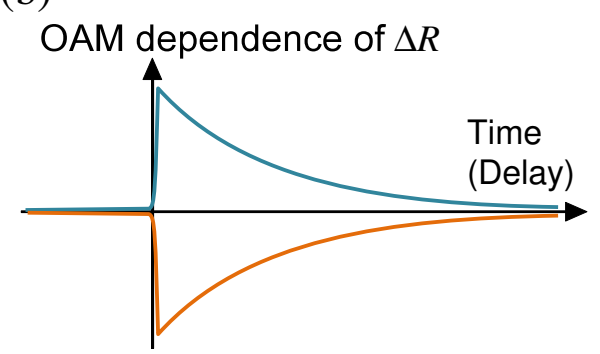

Fig. 1 Schematic illustrations of the OAM dynamics in a timeresolved Pp spectroscopy. (a) OAM distributions for $\ell= \pm 1$ at different delay time $(\tau)$. Nonequilibrium carrier densities between different chiral states are induced by the pump excitation with an OAM (e.g., $\ell=+1$ ) and relax to the equilibrium. (b) Transient reflectivity changes $\Delta R$ with different OAM, which can be resolved by the time-delayed probe pulses with OAM $(\ell= \pm 1)$.

Bi2212 and successfully obtain the non-equilibrium OAM dynamics below $T_{\mathrm{c}}$, indicating the chiral symmetry breaking of the SC state induced by the optical vortex. The comparison with the QP dynamics with the standard Pp spectroscopy suggests that the OAM dynamics reflects the delocalized OAM associated with the SC with long range order.

\section{Experiments}

In laser spectroscopy, the optical vortex transfers its OAM to the electrons through local and delocalized interactions[1416]. The local OAM is dominated in the atom-like quantized system, where the OAM of the vortex beam extends the selection rules for the optical transition[17]. On the other hand, for the high energy non-resonant photoexcitation in metals or superconductors, the delocalized OAM, namely the QP excitation with an azimuthal momentum flow within the photoexcited area, essentially contributes to the signal. Figure 1 shows schematic illustrations of the transient OAM dynamics induced by the pump pulses with an OAM. Hereafter we consider the simple case of QP momentum distributions with different chirality $(\ell= \pm 1)$. In analogy to the case of the pump-induced transient polarization anisotropy, the OAM dynamics is reflected by the pump-induced QP densities with different OAM chirality. Unlike the polarization spectroscopy, the change of the chiral symmetry induced by the pump occurs only when the system contains long- (a)
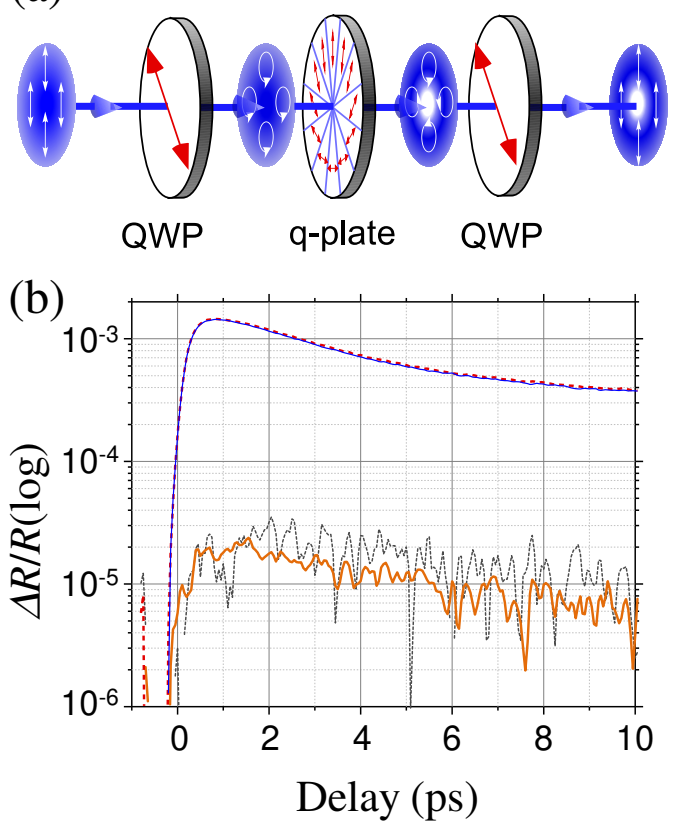

Fig. 2 (a) A schematic illustration of the transverse mode conversion using a q-plate and a pair of quarter wave plates (QWPs) for generating an optical vortex with $\ell=+1$, where the q-plate is a half wave plate with an azimuthally varying orientation of the fast axis (red arrows). (b) Transient $\Delta R$ (pump, probe) at $10 \mathrm{~K}$ with $\mathscr{F} \approx \mathscr{F} S C=1$ $\mu \mathrm{J} / \mathrm{cm}^{2}$. Intense signals were $\Delta R(\perp)=\left(\Delta R\left(\ell^{+}, \ell^{-}\right)+\Delta R\left(\ell^{-}, \ell^{+}\right)\right) / 2$ (red dashed line) and $\Delta R(\|)=\left(\Delta R\left(\ell^{+}, \ell^{+}\right)+\Delta R\left(\ell^{-}, \ell^{-}\right)\right) / 2$ (blue thin line), respectively. Both signals are obtained by the standard $\mathrm{Pp}$ with fluence modulation, where $\ell^{ \pm}= \pm 1$. Black dotted line indicates the OAM transient $\Delta R(\perp)-\Delta R(\|)$ obtained from the difference of above 2 curves. Thick orange line is $\Delta R(\perp)-\Delta R(\|)$ obtained from OAM modulation.

range order because the momentum flow is governed by the photoexcited area. The chiral symmetry change is thus measured by the OAM dependence of the time-delayed probe pulse that is spatially overlapped with the pump-excited area in the sample.

The sample studied was an UD Bi2212 single crystal with $T_{\mathrm{c}} \approx 69 \mathrm{~K}$ grown by the traveling solvent floating zone method. The Pp measurements were performed using a 120 fs pulse train from a cavity-dumped mode-locked Ti:sapphire oscillator for the probe (at $800 \mathrm{~nm}$ ) and its frequency-doubled pulse train for the pump (at $400 \mathrm{~nm}$ ) with a repetition rate of $380 \mathrm{kHz}$. The Gaussian-like pump and probe pulses were independently converted to the optical vortex pulses by using the transverse mode conversion technique described below. Both pulses were coaxially overlapped by a dichroic mirror and focused onto the $a b$-plane of the crystal, allowing us to precisely evaluate the OAM dynamics.

To create the optical vortex pulses, we used our original conversion technique using a q-plate sandwiched between two quarter wave plates (QWPs) as shown in Fig. 2 (a), where the linearly polarized Gaussian optical pulses 
are first converted into the circularly polarized pulses by the QWP and are then into the vortex pulses by the q-plate; the circularly polarized vortex pulses is finally converted back to the linearly polarized vortex pulses after passing through another QWP. It is important to note that the polarization is unchanged in this process. The azimuthally rotated fast axis in the q-plate provides an azimuthal rotating phase difference, generating optical vortex pulses with a rotational phase, where the chirality of the output OAM is determined by the direction of circularly polarized Gaussian pulses. Therefore the optical vortex with opposite chirality can be generated by simply changing the orientation of the QWP. When performing the OAM modulation spectroscopy, we modulated the OAM of the pump between $\ell=+1$ and 1 at around $30 \mathrm{~Hz}$ in order to use lock-in detection. Here we used a pair of liquid crystal variable retarders (LCVs) instead of QWPs for the electric control. The qualities of OAM of the pump and probe pulses were checked by the astigmatic transformation method[18].

\section{Results and discussions}

The intense signals indicated by thin (blue) and dashed (red) lines in Fig. 2 (b) show the $\Delta R$ obtained by using a fluence modulation, where the pump pulses were chopped by an optical chopper. The signals were obtained by the crossed and parallel configurations of the OAM for the pump and probe pulses at $T=10 \mathrm{~K}$. At this temperature, the QP dynamics associated with the $\mathrm{SC}$ is dominant and shows an intense $\Delta R[19]$. The crossed configuration $\Delta R(\perp)$ indicates the chirality of pump and probe OAM are opposite while the parallel $\Delta R(\|)$ indicates both are equal. Here, in order to reduce the contribution of the QP population difference, we evaluated the average of $\Delta R$ with different configurations obtained by using optical vortex pump with OAM of \pm 1 . Although the signal in each configuration is almost identical, a small difference can be confirmed by the difference of $\Delta R$ shown by the dotted line (black).

The signal to noise ratio of the difference signal was significantly improved by using the OAM/chiral modulation of the pump as shown by the thick line (orange). Here we modulate the OAM of the pump with a fixed fluence by modulating the retardation of LCVs as mentioned above. These results clearly indicate the OAM dependence of the transient signal, and suggests the chiral symmetry of the SC is broken by the optical OAM-induced QP excitation. More importantly, the magnitude of the transient OAM in the crossed configuration is larger than that in the parallel one. This implies that the photoexcited QPs with OAM enhance the SC component with opposite chirality and/or suppress the component with equal chirality, suggesting that the OAM transient signal reflects the change in the SC state more effectively than that in the QP state.
In Fig. 3, we compare the temperature dependences of the OAM and QP dynamics across $T_{\mathrm{c}}$. Here we evaluated the transient OAM signal observed by one of the two OAM configurations $\left(\Delta R\left(\perp: \ell^{+}, \ell^{-}\right)-\Delta R\left(\|: \ell^{-}, \ell^{-}\right)\right)$. In this case, however, an instantaneous signal due to the QP population inversion appears in the vicinity of zero delay (just after the pump excitation). Except for this artifact, we confirmed that the signals are compatible with the OAM dynamics. The upper figures plot the signal amplitudes as a function of temperature. In the OAM dynamics, we estimated the signal amplitude by removing the artifact. The temperature dependences of the magnitudes for the OAM and QP dynamics show a similar trend and is consistent with the previous work[20]. However, around $T_{\mathrm{c}}$, the QP dynamics shows a significant amount of signal, which remains even well above $T_{\mathrm{c}}$ while the OAM dynamics shows virtually no signal. To verify the absence of the OAM signal above $T_{\mathrm{c}}$, we plot the transient signal at around $T=80 \mathrm{~K}$ in Fig. 3 (c) together with the QP dynamics in Fig. 3 (d). The OAM dynamics is a little bit noisy, but there is almost no signal, which is contrary to the distinct feature of the SC component in the QP dynamics. The SC component observed in QP dynamics is associated with the pairing fluctuation above $T_{\mathrm{c}}$ because the QP population obtained from the standard Pp measurement is sensitive to the pairing amplitude[20]. In another words, the QP dynamics show no information of the SC phase coherence. In contrast, the OAM dynamics shows virtually no signal above $T_{\mathrm{c}}$, which is consistent with that the nonequilibrium OAM dynamics reflects the delocalized OAM with long range order and is thus sensitive to the SC phase coherence.

We further comment on the difference between the OAM and QP transient dynamics. In Fig. 2 (b), the OAM dynamics $(\Delta R(\perp)-\Delta R(\|))$ is distinct from the QP dynamics $(\Delta R(\perp$ or $\|)$ ). Indeed, the relaxation time of the OAM $(\approx 6.6 \mathrm{ps})$ is estimated to be longer than the QP relaxation time of 2.5 ps. The difference may be reasonable if the OAM transient is dominated by the SC coherence while the QP reflects the pairing amplitude. Here we note that the OAM signal was very sensitive to the spatial overlap between the pump and probe OAM pulses.

\section{Summary}

The OAM resolved pump-probe spectroscopy using optical vortex pulses was performed on UD-Bi2212 superconductor. The pump OAM induced $\Delta R$ shows a small difference depending on the probe OAM (OAM dynamics), which can be uniquely resolved by using a novel OAM modulation spectroscopy. The magnitude of the transient OAM in the crossed OAM chilarity is larger than that in the parallel OAM chilarity, indicating that the photoexcited QPs 

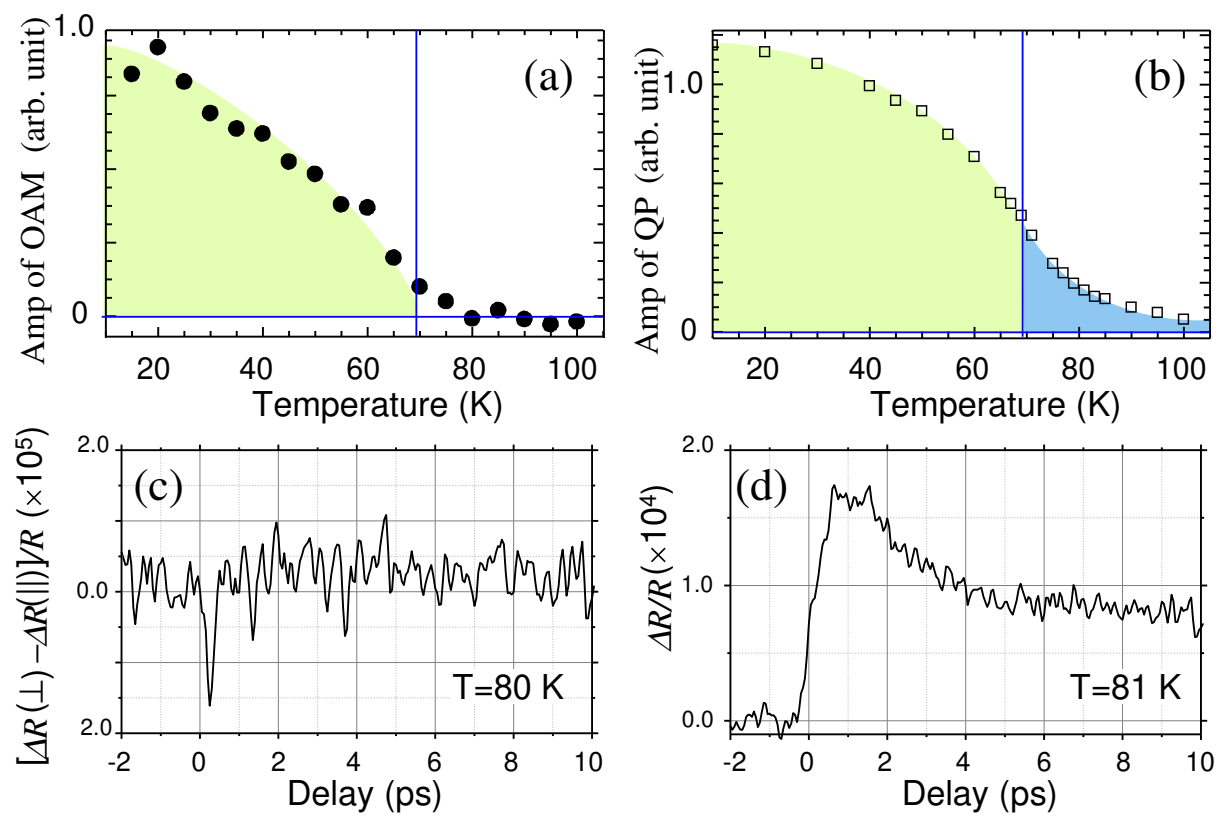

Fig. 3 Plots of the amplitude of the (a) OAM transient $(\Delta R(\perp)-\Delta R(\|))$ and (b) QP transient $(\Delta R)$ responses as a function of temperature. The solid lines indicate the $T_{\mathrm{c}} \approx 69 \mathrm{~K}$. To highlight the difference of the temperature dependences, the signals below and above $T_{\mathrm{c}}$ are shaded in different colors. Typical transient (c) OAM dynamics and (d) QP dynamics obtained at the temperature above $T_{\mathrm{c}}$. In (c), the impulsive response around zero delay is an artifact due to the QP population inversion, which is removed when the signal amplitude is estimated in (a).

with OAM enhance the SC component with opposite chirality and/or suppress the component with equal chirality. The temperature dependence of the OAM dynamics shows the absence of the pairing fluctuation, suggesting that the OAM dynamics is sensitive to the delocalized OAM associated with long range order.

\section{References}

1. C. Tsuei, J. Kirtley, Reviews of Modern Physics 72(4), 969 (2000)

2. B. Keimer, S. Kivelson, M. Norman, S. Uchida, J. Zaanen, Nature 518(7538), 179 (2015)

3. M. Vershinin, S. Misra, S. Ono, Y. Abe, Y. Ando, A. Yazdani, Science 303(5666), 1995 (2004)

4. C. Howald, H. Eisaki, N. Kaneko, M. Greven, A. Kapitulnik, Physical Review B 67(1), 014533 (2003)

5. Y. Kohsaka, C. Taylor, K. Fujita, A. Schmidt, C. Lupien, T. Hanaguri, M. Azuma, M. Takano, H. Eisaki, H. Takagi, et al., Science 315(5817), 1380 (2007)

6. K. Lang, V. Madhavan, J. Hoffman, E. Hudson, H. Eisaki, S. Uchida, J. Davis, Nature 415(6870), 412 (2002)

7. T. Kurosawa, T. Yoneyama, Y. Takano, M. Hagiwara, R. Inoue, N. Hagiwara, K. Kurusu, K. Takeyama, N. Momono, M. Oda, M. Ido, Phys. Rev. B 81, 094519 (2010). DOI 10.1103/PhysRevB.81.094519. URL https://link.aps.org/doi/10.1103/PhysRevB.81.094519

8. J. Tranquada, B. Sternlieb, J. Axe, Y. Nakamura, S. Uchida, Nature 375(6532), 561 (1995)

9. M. Fujita, H. Goka, K. Yamada, M. Matsuda, Physical review letters 88(16), 167008 (2002)

10. J. Orenstein, J.E. Moore, Physical Review B 87(16), 165110 (2013)

11. H. Karapetyan, M. Hücker, G. Gu, J. Tranquada, M. Fejer, J. Xia, A. Kapitulnik, Physical review letters 109(14), 147001 (2012)
12. Y. Toda, F. Kawanokami, T. Kurosawa, M. Oda, I. Madan, T. Mertelj, V.V. Kabanov, D. Mihailovic, Phys. Rev. B 90, 094513 (2014). DOI 10.1103/PhysRevB.90.094513. URL https://link.aps.org/doi/10.1103/PhysRevB.90.094513

13. L. Allen, M.W. Beijersbergen, R.J.C. Spreeuw, J.P. Woerdman, Phys. Rev. A 45, 8185 (1992). DOI 10.1103/PhysRevA.45.8185. URL https://link.aps.org/doi/10.1103/PhysRevA.45.8185

14. Y. Ueno, Y. Toda, S. Adachi, R. Morita, T. Tawara, Optics express 17(22), 20567 (2009)

15. Y. Tokizane, K. Shimatake, Y. Toda, K. Oka, M. Tsubota, S. Tanda, R. Morita, Optics express 17(26), 24198 (2009)

16. M.A. Noyan, J.M. Kikkawa, Applied Physics Letters 107(3), $032406(2015)$

17. C.T. Schmiegelow, J. Schulz, H. Kaufmann, T. Ruster, U.G. Poschinger, F. Schmidt-Kaler, Nature communications 7 (2016)

18. V. Denisenko, V. Shvedov, A.S. Desyatnikov, D.N. Neshev, W. Krolikowski, A. Volyar, M. Soskin, Y.S. Kivshar, Optics express 17(26), 23374 (2009)

19. Y. Toda, T. Mertelj, P. Kusar, T. Kurosawa, M. Oda, M. Ido, D. Mihailovic, Phys. Rev. B 84, 174516 (2011). DOI 10.1103/PhysRevB.84.174516. URL https://link.aps.org/doi/10.1103/PhysRevB.84.174516

20. I. Madan, T. Kurosawa, Y. Toda, M. Oda, T. Mertelj, P. Kusar, D. Mihailovic, Scientific reports 4, 5656 (2014) 